Parkinson's disease can employ a predictive motor strategy. $J$ Neurol Neurosurg Psychiatry 1984;47:1299-306.

${ }^{2}$ Flowers KA. Lack of prediction in the motor behavior of Parkinsonism. Brain 1978; 101:35-52.

${ }^{3}$ Stern Y, Mayeux R, Rosen J, Ilson J. Perceptual motor dysfunction in Parkinson's disease: $\mathbf{A}$ deficit in sequential and predictive voluntary movement. J Neurol Neurosurg Psychiatry 1983;46:145-51.

${ }^{4}$ Stern Y, Mayeux R, Rosen J. Contribution of perceptual motor dysfunction to construction and tracing disturbances in Parkinson's disease. J Neurol Neurosurg Psychiatry 1984;47:983-94.

${ }^{5}$ Bloxham CA, Mindel TA, Frith CD. Initiation and execution of predictable and unpredictable movements in Parkinson's disease. Brain 1984;107:371-84.

Day et al reply

We thank Dr Stern for his comments and suggestions, but still believe that our conclusions hold. As stated in the final paragraph of the discussion to our paper, we think that the data show that patients with Parkinson's disease can and do adopt a predictive motor strategy when tracking a known target movement, but that they do so with less accuracy than normal subjects.

The issue of short-term prediction of unknown targets, which leads to tracking lags shorter than visual reaction times in both normals and Parkinsonians, is not the crucial point. It is the ability of Parkinsonians to reduce tracking lag closer to zero when the target movement is known that defines the use of a predictive motor strategy, and 11 of the 12 patients studied were able to do this. If, as suggested by Dr Stern, the patients and controls are compared by measuring the reduction in lag for each subject, then a $t$ test does reveal a difference between the groups at the $5 \%$ level of significance $(t=2.29, \mathrm{p}=0.33$ for a twotailed test). ANOVA gives essentially the same result with a significant main effect of conditions $(F=46.88, p<0.001)$ but not of groups $(\mathrm{F}=0.05, \mathrm{p}>0.05)$ with a significant interaction between groups and conditions $(F=5 \cdot 24, p=0.034)$. However, it should be pointed out that strictly speaking a $t$ test is not applicable to these data. The difference in variability of the two groups is large (variance ratio $=6.24$ ) and the control data is skewed. The nonparametric Mann-Whitney test is more suitable but fails to reveal a difference between the groups $(U=23, p>0.05)$. Therefore, we believe that the present data are inconclusive on this point, and that this issue can be resolved only by further experimentation.
Our data show that even though a predictive strategy was used, the tracking error of the patient group did not improve as much as the control group. There are two possible ways of explaining this. Either the patients' tracking lag did not improve as much as controls', or, lag was reduced normally but the movements were more inaccurate. In contrast to Dr Stern, we favour the latter explanation for the following reasons: (1) The mean tracking lag of the patients was not statistically different from that of the controls in both the unpredictable and predictable conditions. (2) There was a correlation between the improvement in error and reduction in lag for the control subjects $(r=0.83, p<0.05)$, but not for the patient group $(r=0.03, p>0.05)$.

In conclusion, Dr Stern and we agree that patients with Parkinson's disease can adopt predictive movement, but less successfully than normal subjects.

\section{Triphasic waves of metabolic encephalopathy versus spike-wave stupor}

Sir: We disagree with the discussion that Yamamoto and Hosokawa give in their recent case report "Triphasic spike-wave stupor in portal-systemic encephalopathy."1 They seem to confuse the EEG findings of triphasic waves seen in metabolic disturbances and spike-wave discharges seen in epileptic spike-wave stupor (also called absence status or petit mal status). This distinction has diagnostic and therapeutic importance.

Their patient was a 59-year-old woman presenting with episodic obtundation and a flapping tremor. Portal-systemic encephalopathy was diagnosed from laboratory and radiologic evidence of liver cirrhosis (including hyperammonaemia) and treated with lactulose and kanamycin. They concluded from EEG findings of "continuous bilaterally synchronous atypical spike-wave discharges of an epileptic nature" that the patient had spike-wave stupor secondary to portal-systemic encephalopathy.

A more accurate interpretation of the EEG (shown in the figure accompanying the case report) would be triphasic waves suggestive of a metabolic disturbance. Triphasic waves are medium to high amplitude, diffuse, frontally predominant, broad complexes consisting of a large positive (downward) sharp wave preceded and followed by small negative (upward) components. ${ }^{2}$ These complexes last 0.15 to 0.25 seconds and recur singly or in serial trains about every 0.5 to 2 seconds. They are often super- imposed on a background of asynchronos and bisynchronous slow waves. There is usf ally a tendency for fronto-occipital delaq. Although rarely seen in degenerative pro cesses or after anoxic episodes and hesd trauma, triphasic waves usually indicafte toxic-metabolic encephalopathy, most com monly hepatic or renal. They are not exleptic phenomena. ${ }^{3}$ There appears to be $\exists$ limited correlation between blood ammon levels and the triphasic wave pattern. ${ }^{4}$ The treatment is usually correction of the undeflying metabolic abnormality.

The spike-wave discharges accompanyin spike-wave stupor are medium to high amplitude, generalised, predominanfy fronto-midline, synchronous complexes (surface negative spike followed by surface negative slow wave). The morphology mépy be less regular than the spike-wave of typica absence seizures, and polyspikes may be admixed. ${ }^{5}$ These repetitive spike-wave copbj plexes fire rhythmically at a frequency of $2 \$$ $6 \mathrm{~Hz}$ (usually $2.5-3.5 \mathrm{~Hz}$ ). ${ }^{6}$ The complexes may be continuous, or they may occur repetitive bursts alternating with normal कo near-normal background activity. A strong external stimulus may temporarily thö malise the EEG. ${ }^{5}$

Spike-wave stupor (absence status) epileptic phenomenon clinically manifeging as a confused state lasting minutes to dey ${ }^{8}{ }^{8}$ In most instances, it occurs as part of a p existing seizure disorder, usually absenoe se్ zures. $^{56}$ The treatment is usually by aniepileptic medications. ${ }^{9}$

This case report illustrates how an finding (triphasic waves) combined wfth appropriate laboratory and radiologre confirmation can differentiate the diagnogs of portal-systemic encephalopathy froin other causes of episodic obtundation, including spike-wave stupor. Yamamofo and Hosokawa correctly point out that spike-wave stupor has occasionally be $\overrightarrow{\mathrm{gn}^{n}}$ reported in patients without a previous seizure history. Rarely, the condition may be associated with acquired neurological d ease (head injury, encephalitis, cerebrovascular disease) ${ }^{6}$ or predisposing tox $\overline{\text { e }}-$ metabolic disturbances. ${ }^{10}$ The same associations occur with typical absence seizures. $^{9}$

The EEG distinction between triphase waves and spike-wave discharges makes EEG useful in ensuring that metabolic diserders and epileptic spike-wave stupor ałe each diagnosed and treated appropriatel

PHILIP H SHERID $\overline{\overline{A N}}$ SUSUMU SAФO

National Institute of Neurologinal 